\title{
New CMAJ policy on sharing data from clinical research
}

\author{
John Fletcher MB BChir MPH
}

$\mathrm{B}$ eginning Apr. 1, 2014, CMAJ will require authors of clinical trials of drugs and medical devices to include a data-sharing statement in their manuscripts; however, we will not require authors to agree to share their data, regardless of the study design. We will welcome data-sharing statements for all research articles, but statements will be mandatory only for clinical trials. In their statements, authors should indicate whether any, all or portions of the data are available to others; where, through whom, when and on what terms data will be available; and how data may be accessed.

Some medical journals, such as BMJ and PLoS Medicine, have encouraged data sharing for several years, and last year $B M J$ made data sharing a condition of publication for trials. $C M A J$ is adopting a voluntary policy. Why? We know that many researchers are pleased to collaborate in a transparent way and are willing to allow others access to their data. For them, no compulsion is needed. However, two groups have a strong interest to resist initiatives to share data widely. First, those who sell a product or service and have conducted research stand to lose sales if they disclose information that is unfavourable. Drug companies have argued strongly that data from clinical trials are commercial in confidence, and many companies have resisted calls to make their data more widely available. Second, some researchers, who must "publish or perish," and rely on their data as a resource for several of their articles stand to lose publishing opportunities if they disclose all of their data before they have found the time or resources to complete all of their planned publications.

Because the two groups with the most to lose are the same groups who currently have control of the data, meaningful change will not take place without first addressing their needs. Researchers can protect their interests by paying close attention to data definitions and timing. For example, researchers could release restricted datasets that are relevant only to reported analyses at the time of publication and release the full dataset within a few years. For the manufacturers of drugs and devices the problem is thornier. Companies pay for research as part of the licensing process and may claim ownership of the information produced. However, society demands access to this research through product licensing regulations, and participants in research usually take part in trials believing they are contributing to a common good and not just to a company's bottom line. Regulators or other large organizations must address these competing interests.

The Nordic Cochrane Centre and $B M J$ have fuelled the present interest in data sharing by asking for full disclosure of clinical trial data surrounding the effectiveness of Tamiflu (oseltamivir), a drug that has been stockpiled in large quantities in developed countries as part of emergency planning for a possible influenza pandemic. ${ }^{1}$ Despite their best efforts to obtain data, researchers say that about $60 \%$ of the trial data concerning Tamiflu remains hidden. ${ }^{2}$

Encouraging open sharing of data on a voluntary basis has its own benefits, even if it cannot directly combat intentional concealment. Data sharing could provide answers to the following questions: Did they get it right? Did they miss anything important? Can we use these data to learn more?

Ensuring the credibility of published research is the central focus of academic peer review, yet this process is a notoriously poor detector of error or fraud. Existing editorial policies allow editors to ask authors for their original data as part of the peer review process. However, an individual paper is often reviewed by only a handful of people before publication. Extending the scrutiny of the underlying data into the postpublication period is a logical step.

Sharing data may also benefit society by allowing new knowledge to be generated in ways that would not be possible without cooperation. For example, the effects of interventions are seldom uniform and may vary across different populations of patients. The analyses to detect such subgroup effects and to enable physicians to personalize treatment options require large amounts of data. Without access to individual patient data, such analyses are impossible.

Fortunately, there are early adopters who are already embracing the practice of data sharing. Data from the Canadian Community Health Survey are available to any Canadian researcher with a reasonable research question and an analytic plan. GlaxoSmithKline has an online registry of all its trials and says it will allow scientists to request access to anonymized patient-level data. ${ }^{2}$ Researchers who wish to share their data may use websites such as www.datadryad.org to do so. By joining these early adopters, CMAJ hopes to encourage Canadian researchers to embrace a new norm, so society can check that they did the right thing, see that nothing has been missed and ask to use the data to generate new knowledge.

\section{References}

1. Tamiflu campaign. BMJ 2014. Available: www.bmj.com/tamiflu (accessed 2014 Jan. 13)

2. Collier R. UK Parliament calls for sharing of all clinical trial data. CMAJ 2014; Jan. 13 [Epub ahead of print].

Competing interests: See www.cmaj.ca/site/misc/cmaj_staff.xhtml

Affiliation: John Fletcher is Editor-in-Chief, CMAJ.

Correspondence to: $C M A J$ editor, pubs@cmaj.ca

CMAJ 2014. DOI:10.1503/cmaj.140041 